\title{
Vaccine-Associated Uveitis
}

by Matthew Benage, BS \& Frederick W. Fraunfelder, MD

\begin{abstract}
While uveitis may be a rare adverse effect of vaccines, awareness of this potential reaction to vaccines is important, as is reporting such occurrences to adverse drug reaction registries.
\end{abstract}

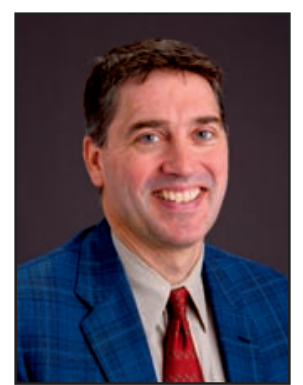

Matthew Benage, BS, is a fourth-year medical student at the University of Missouri School of Medicine. Frederick W. Fraunfelder, MD, MBA, (above), MSMA member since 2014, is chairman and the Roy E. Mason and Elizabeth Patee Mason Distinguished Professor of the Department of Ophthalmology, Mason Eye Institute, at the University of Missouri. Dr. Fraunfelder is director of the National Registry of Drug-Induced Ocular Side Effects (www. eyedrugregistry.com).

Contact: fraunfelderf@health.missouri.edu
Abstract

All of the widely administered vaccines have been reported to cause uveitis. The ocular inflammation is usually temporary and resolves with topical ocular steroids. During a 26-year period, a total of 289 cases of vaccine-associated uveitis were reported to three adverse reaction reporting databases. Hepatitis B vaccine, either alone or administered with other vaccines, appears to be the leading offender. Clinicians are encouraged to report cases of vaccine- or drug-associated ocular adverse reactions to www.eyedrugregistry.com

\section{Introduction}

Millions of lives have been saved since Edward Jenner's pioneering work, in 1796, to create a smallpox vaccine. ${ }^{1}$ Disease prevention by means of vaccination is one of public health's greatest successes., ${ }^{2,3}$ But the benefits of vaccines also carry the potential for a wide variety of side effects. Cases of uveitis in association with vaccine administration have been reported with nearly all vaccines. ${ }^{4,5}$

To examine the occurrence of vaccine-associated uveitis, we collected data on cases of vaccineassociated uveitis reported to three spontaneous reporting databases on adverse drug events.

\section{Methods}

Spontaneous reports of all cases of vaccine-associated uveitis, made from 1984 to 2014, were collected from the National Registry of Drug-Induced Ocular Side Effects (www.eyedrugregistry.com), the World Health Organization (WHO) Monitoring Centre (Uppsala, Sweden), and the FDA spontaneous reporting system (Bethesda, MD). In addition, a Medline literature search was performed using the keywords of uveitis, iritis, iridocyclitis, or vitritis, in combination with vaccines, hepatitis A, hepatitis B, HPV, BCG, brucella, DPT, herpes, influenza, measles, MMR, pneumococcal, smallpox, tetanus, typhoid, heine medin, varicella, yellow fever, and zoster vaccine.

\section{Results}

A total of 289 cases of vaccineassociated uveitis were reported between 1984 and 2014. A

preponderance of cases occurred in females: 199 cases in females versus 77 in males. Gender was not reported in 13 cases. Mean age of uveitis cases was 30 years (age range: 2 months to 86 years). The median number of days between vaccination and onset of uveitis was 16 days (range: 1 day to 6 years; $\mathrm{SD}=362$ days). 
Table 1

Data on 289 Reported Cases of Vaccine-Associated Uveitis, 1984-2014

\begin{tabular}{|c|c|c|c|}
\hline Vaccine & Number of cases & Average age (range) & Outcome \\
\hline Bacille Calmette-Guerin & $21-11 \mathrm{~F}, 7 \mathrm{M}, 3$ unknown & 60 years ( $13-85$ years) & $\begin{array}{l}6 \text { recovered, } 3 \text { unresolved, } 12 \\
\text { unknown }\end{array}$ \\
\hline Brucella & $1-$ unknown & Unknown & Unknown \\
\hline DPT & $1-1 \mathrm{~F}$ & 38 years & Unknown \\
\hline Hepatitis A (alone) & $5-4 \mathrm{~F}, 1 \mathrm{M}$ & 48 years ( $11-78$ years) & 3 recovered, 2 unknown \\
\hline Hepatitis B (alone) & $115-74 \mathrm{~F}, 39 \mathrm{M}, 1$ unknown & 29 years ( $<1$ mon to 58 years) & $\begin{array}{l}52 \text { resolved, } 9 \text { unresolved, } 54 \\
\text { unknown }\end{array}$ \\
\hline Herpes zoster & $1-1 \mathrm{M}$ & 59 years & Unknown \\
\hline HPV & $44-44 \mathrm{~F}$ & 17 years (11-29 years) & $\begin{array}{l}10 \text { recovered, } 1 \text { unresolved, } 33 \\
\text { unknown }\end{array}$ \\
\hline Influenza & $28-17 \mathrm{~F}, 8 \mathrm{M}, 3$ unknown & 55 years (14-82 years) & $\begin{array}{l}9 \text { recovered, } 2 \text { unrecovered, } 1 \\
\text { sequelae, } 16 \text { unknown }\end{array}$ \\
\hline Measles & $1-1 \mathrm{M}$ & 10 years & Unknown \\
\hline MMR & $13-8 \mathrm{~F}, 4 \mathrm{M}, 1$ unknown & 7 years (<1-17 years) & $\begin{array}{c}1 \text { recovered, } 1 \text { recovering, } 2 \\
\text { unrecovered, } 9 \text { unknown }\end{array}$ \\
\hline Pneumococcal & $1-1 \mathrm{~F}$ & 67 years & Unknown \\
\hline Pox viral particles & $1-$ unknown & Unknown & Unknown \\
\hline Smallpox & $2-2 \mathrm{~F}$ & 25 and 34 years & 1 recovered; 1 unknown \\
\hline Tetanus & $1-1 \mathrm{~F}$ & 50 years & 1 recovered \\
\hline Heine Medin & $1-$ unknown & Unknown & Unknown \\
\hline Varicella & $13-7 \mathrm{~F}, 6 \mathrm{M}$ & 27 years (4.8-86 years) & 2 recovered, 11 unknown \\
\hline Unknown vaccine & $2-2 \mathrm{~F}$ & 27 and 68 years & Unknown \\
\hline \multicolumn{4}{|c|}{ SIMULTANEOUSLY ADMINISTERED VACCINES (SEE TABLE 2 FOR SPECIFIC VACCINE COMBINATIONS) } \\
\hline $\begin{array}{l}\text { DPT and vaccine(s) other than } \\
\text { hepatitis B }\end{array}$ & 3 & Unknown & Unknown \\
\hline Hepatitis A and B combined & 10 & Unknown & Unknown \\
\hline $\begin{array}{l}\text { Hepatitis A and vaccine(s) other } \\
\text { than hepatitis B }\end{array}$ & 4 & Unknown & Unknown \\
\hline $\begin{array}{l}\text { Hepatitis B and vaccine(s) other } \\
\text { than hepatitis A }\end{array}$ & 11 & Unknown & Unknown \\
\hline HPV and other vaccine(s) & 4 & Unknown & Unknown \\
\hline MMR and varicella & 2 & Unknown & Unknown \\
\hline Typhoid and polio & 1 & Unknown & Unknown \\
\hline
\end{tabular}

Table 1 lists the vaccines associated with uveitis and the number of cases reported with each vaccine. Vaccines implicated in the case reports of uveitis included bacilli Calmette-Guerin (BCG), brucella, diphtheria-pertussistetanus (DPT), hepatitis A, hepatitis B, herpes zoster, human papillomavirus (HPV), influenza, measles, measlesmumps-rubella (MMR), pneumococcal, pox viral particles, smallpox, tetanus, Heine-Medin, and varicella. Thirty-five of the 289 cases of uveitis were associated with simultaneous administration of more than one vaccine (see Table 2 for the list of vaccine combinations and reported cases of uveitis).

Vaccines most commonly reported in association with uveitis were as follows, in descending order of frequency: hepatitis B vaccine, 115 cases; HPV vaccine, 44 cases; influenza vaccine, 28 cases; $\mathrm{BCG}$ vaccine, 21 cases; and MMR vaccine and varicella vaccine, 13 cases each.
Information on which dose of a particular vaccine was associated with uveitis was not available in the reporting systems.

\section{Hepatitis $B$ Vaccine}

The data point to hepatitis B vaccine as the leading offender for causing uveitis. A total of 115 cases (40\%) of uveitis were reported in association with hepatitis $B$ vaccine alone. Seventy-four cases were in females, 39 in males, and gender was not specified in 1 case. Mean age of was 29 years (age range: 1 month to 58 years). The median interval between vaccine administration and onset of uveitis was 23 days (range: 1 day to 6 years). Resolution of uveitis was reported in 52 cases, no resolution in 9 cases, and the outcome was not reported in 54 cases. The median duration of uveitis was 346 days (range: 31-686 days). 
Table 2

\section{Breakdown of Reported Cases of Uveitis in Association with Simultaneously Administered Vaccines}

\begin{tabular}{|c|c|}
\hline Vaccine combination & $\begin{array}{l}\text { Number of } \\
\text { cases }\end{array}$ \\
\hline DPT/Meningococcal & 1 \\
\hline DPT/Pertussis & 1 \\
\hline DPT/Pneumococcal & 1 \\
\hline Hep A/Polio/Typhoid & 1 \\
\hline Hep A/Varicella & 1 \\
\hline Hep A/Hep B & 10 \\
\hline Hep A/Typhoid & 1 \\
\hline Hep A/Yellow Fever & 1 \\
\hline Hep B/DPT & 2 \\
\hline Hep B/DPT/polio & 1 \\
\hline Hep B/Tetanus/Typhoid/Yellow Fever & 1 \\
\hline Hep B/Tuberculin & 1 \\
\hline Hep B/Typhoid & 6 \\
\hline HPV/Tetanus & 2 \\
\hline HPV/MMR/Varicella & 2 \\
\hline MMR/Varicella & 2 \\
\hline Typhoid/Polio & 1 \\
\hline
\end{tabular}

In contrast to the 115 cases of uveitis related hepatitis $B$ vaccine, 5 cases were reported with hepatitis A vaccine. Four occurred in females and 1 in a male. Mean age was 48 years (range: 11-78 years). The median interval between vaccination and onset of uveitis was 5 days (range: 2-30 days). Recovery occurred in 3 cases, and resolution did not occur in 2 cases.

\section{Human Papillomavirus Vaccine}

Of the 289 reported cases of vaccine-associated uveitis, 44 (15\%) were reported in association with HPV vaccine. All cases were female, with an average age of 17 years (age range: 11-29 years). The median time to onset of uveitis after vaccination was 30 days (range: 2-511 days). Recovery was reported in 10 cases, lack of recovery in 1 case, and the outcome was not reported in 33 cases.

\section{Influenza Vaccine}

A total of 28 cases of uveitis were reported following administration of the influenza vaccine. Cases occurred in 17 females and 8 males, and gender was not specified in 3 cases. The average age was 55 years (age range: 14-82 years). The median time to onset of uveitis was 1 day (range: 1-92 days). Nine cases were reported to have recovered, 1 case recovered with sequelae, 2 cases were unresolved, and in 16 cases the outcomes were unknown.

\section{Bacille Calmette-Guerin Vaccine}

Twenty-one cases of uveitis were reported following the administration of the BCG vaccine. Eleven of the cases occurred in females, 7 occurred in males, and gender was not reported in 3 cases. Mean age was 60 years (age range: 13-85 years). Data were insufficient to report median interval between BCG vaccination and onset of uveitis. The median duration of uveitis was 72 days (range: 35-514 days). Six patients were reported to have recovered, 3 patients were recovering, and outcome was not reported in 12 cases.

\section{Measles-Mumps-Rubella Vaccine}

Thirteen cases ( 8 females, 4 males, 1 unknown) of uveitis were reported following administration of MMR vaccine. The average age was 7 years (age range: 0.9-17 years). The median days to onset were 21 (range: 3-145 days). One patient was reported to have recovered, 1 patient is recovering, 2 have not recovered, and no outcome data were reported in 9 patients.

\section{Varicella Vaccine}

A total of 13 cases ( 7 females and 6 males) of uveitis were reported following varicella vaccination. The average age was 27 years (age range: $4.8-86$ years). The median time to onset of uveitis was 2 days (range: 2-97 days). Two cases were reported as recovered and 12 cases did not include outcome data.

\section{Simultaneous Administration of Multiple Vaccines}

Of the 289 cases of vaccine-associated uveitis, 35 (8\%) were reported to follow simultaneous administration of multiple vaccines (see Table 2 for the breakdown of the number of cases with each vaccine combination). The most common vaccine combination was hepatitis $B$ and hepatitis A, reported in 10 of the 35 cases of uveitis in association with simultaneous administration of multiple vaccines. In 9 additional cases associated with hepatitis $\mathrm{B}$ vaccine, it was paired with DPT (2 cases), tuberculin (1 case) and typhoid (6 cases). Taken together, data on hepatitis B vaccine, given alone (115 cases) or in combination with other vaccines (21 
cases), indicate it was associated in 136 (47\%) of the 289 reported cases of uveitis.

Other vaccine combinations associated with uveitis included hepatitis A vaccine administered with polio and typhoid, with varicella, with typhoid , and with yellow fever, in 1 case each. Varicella vaccine was given simultaneously with MMR and HPV (all three at the same time) in 2 cases and with MMR in 2 cases. DPT vaccine was administered with meningococcal (1), pertussis (1), and pneumococcal (1) vaccines. HPV vaccine was paired with tetanus vaccine in 2 cases. One case of uveitis was reported in association with simultaneous administration of tetanus, diphtheria, polio, and MMR vaccines, and 1 case was reported with simultaneous administration of typhoid and polio vaccines.

\section{Clinical Features of Uveitis}

Uveitis can occur as an isolated disorder, which is likely the case in most vaccine-associated uveitis, or it can arise from numerous other diseases, including various autoimmune conditions, systemic infections, paraneoplastic syndromes and ocular trauma. In many cases of uveitis, an underlying etiology cannot be identified. Uveitis affects the middle layer of tissue in the eye and is classified by anatomic involvement: anterior uveitis, intermediate uveitis, posterior uveitis and panuveitis. Anterior uveitis, by far the most common, involves the iris. The ciliary body (vitreous) is involved in intermediate uveitis; the choroid, retina and optic disk are involved in posterior uveitis, and diffuse involvement of the eye characterizes panuveitis, which includes both the anterior and posterior chambers.

Uveitis is a vision-threatening inflammation and is considered an ophthalmic emergency. Patients typically describe eye redness, eye pain, blurred vision, decreased vision, light sensitivity, and the presence of floaters.

Intermediate uveitis may be manifested symptomatically by a painless decrease in vision and noticeable floaters. The onset of symptoms can be sudden or gradual. Untreated, uveitis can lead to complications such as glaucoma, cataract, optic nerve damage, retinal detachment, and irreversible vision loss.

On ophthalmological examination, slit-lamp examination in anterior uveitis may reveal clumps of white blood cells on the inner corneal surface (keratic precipitates), cells and flare (a haze) in the anterior chamber, and posterior synechiae. With intermediate uveitis, examination may reveal the presence of inflammatory cells over the pars plana ("snowball" effect), evidence of cystoid macular edema and, in some cases, neovascularization of the retinal periphery. Funduscopic evidence of posterior uveitis includes cells in the vitreous humor and white or yellow- white lesions in the retina or underlying choroid, or both. Cystoid macular edema may be seen, as may evidence of optic disk edema, exudative retinal detachment, and retinal vasculitis.

Management of noninfectious uveitis primarily consists of intensive ocular steroid therapy to reduce the inflammation as quickly as possible. This approach would be used in most patients with vaccine-associated uveitis. Prednisolone acetate 1\%, 1 drop every hour while the patient is awake, is typically given for several weeks. Systemic steroids, such as 1 to $2 \mathrm{mg} / \mathrm{kg}$ of prednisone once daily, may be initiated in severe or chronic uveitis. Other approaches include nonsteroidal immunosuppressive drug therapy for severe or refractory uveitis. Complications of uveitis such as glaucoma, retinal detachment or optic nerve damage, are treated accordingly.

\section{Discussion}

Spontaneous reports often give the first signal that a drug is causing an adverse reaction. They are, however, limited in providing consistent details on the clinical features, clinical course and outcome, and the existence of concomitant disease and other potential contributing factors. The interval between vaccination and the onset of uveitis had a wide range in the reported cases, from a few days to a number of years. Unfortunately, the number of days between vaccination and onset of uveitis was not often reported.

Various hypotheses have been suggested for vaccineassociated uveitis, including molecular mimicry secondary to a close resemblance between vaccine peptide fragments and uveal self-peptides, delayed-type hypersensitivity with deposition of immune complexes, and an immune reaction to vaccination adjuvants. ${ }^{5,6}$ With hepatitis A vaccine, ${ }^{6-8}$ aluminum-containing adjuvant used in the vaccine has been suggested as a cause of an inflammatory response and may be related to uveitis. ${ }^{6-8}$ Our previous study of spontaneous reports of hepatitis $\mathrm{B}$ vaccine-associated uveitis found 32 cases reported between 1982 and 2009 to the National Registry of Drug-Induced Ocular Side Effects, the World Health Organization and the FDA adverse reactions reporting system, the same databases used in the current collection of cases. ${ }^{5}$ (The 32 cases from 1982 to 2009 are included in the current data, since current data encompass the years 1982-2014.)

The explanation for the seemingly disproportionate increase in reported cases of hepatitis B vaccine-associated uveitis, from 32 cases reported in the years 1982-2009 to 136 cases reported in the years from 1982 to 2014, is unknown. Hepatitis B vaccine was approved for use in 1982, and since then hepatitis B prevention strategies have 
evolved to include an increasing number of groups that are recommended to receive the vaccine. From the initial targeted populations that included health care workers and intravenous drug users, hepatitis B vaccine recommendations were expanded in 1991 to universal hepatitis B vaccination in infants, with the first dose administered soon after birth, before discharge from the hospital. Four years later, in 1995, the CDC Advisory Committee on Immunization Practices (ACIP) recommended routine vaccination of all adolescents 11 to 12 years of age who had not been previously vaccination. In 1999, the ACIP recommended that all unvaccinated children under 19 years of age be vaccinated. These strategies indeed reduced hepatitis B transmission in the United States: the incidence of acute hepatitis declined 75\% from 1990 to 2004, with the greatest decline occurring among children and adolescents. ${ }^{9}$

Uveitis from influenza vaccine has been described in various case reports. ${ }^{10,11}$ The mechanism leading to uveitis after influenza vaccination is postulated to be due to inflammation secondary to vaccine additive. ${ }^{12}$ Varicellaassociated uveitis has been documented previously, and the mechanism may relate to reactivation of varicella virus or may be due to inoculum with live attenuated varicella viral particles. ${ }^{13}$

Uveitis has been reported in association with intramuscular injection of live attenuated BCG vaccine for immunotherapy of malignant melanoma and with intravesicular BCG irrigation to treat bladder carcinoma. ${ }^{14,15}$ The hypothesized mechanism for BCG-associated uveitis is molecular mimicry due to the amino acid sequence homology between proteins from BCG and retinal antigens. ${ }^{15}$ Similarly, the mechanism for MMR-associated uveitis has been suggested to involve antigenic mimicry or even faulty preservation of the vaccine. ${ }^{16,17}$

\section{Conclusion}

While uveitis may be a rare adverse effect of vaccines, awareness of this potential reaction to vaccines is important, as is reporting such occurrences to adverse drug reaction registries. That hepatitis $B$ vaccine accounted for a disproportionate number of cases certainly piques curiosity about contributing factors for uveitis in association with hepatitis B vaccine. In a similar vein, we are also left with the question of why a disproportionate number of cases of vaccine-associated uveitis occur in females (199 in females versus 77 in males in our data). Future studies of vaccineassociated uveitis could focus on active surveillance and rigorous clinical evaluation of patients presenting with uveitis temporally related to vaccination.
Dutiful reporting of suspected cases of vaccine- or drugrelated reaction to registries that collect data on adverse events is a key to improving the quality of data tracking of potential adverse reactions from vaccines as well as from drugs and biologic agents. Clinicians who encounter patients with possible ocular side effects to vaccines (or other agents for that matter) are encouraged to report such cases to the National Registry of Drug-Induced Ocular Side Effects (www.eyedrugregistry.com).

\section{References}

1. Stern AM, Markel H. The history of vaccines and immunization: familiar patterns, new challenges. Health Affairs (Project Hope) 2005;24(3):611-621. 2. Strikas RA, Advisory Committee on Immunization Practices (ACIP), ACIP Child/Adolescent Immunization Work Group. Advisory Committee on Immunization Practices recommended immunization schedule for persons aged 0 through 18 years, United States, 2015. Morbid Mortal Wkly Rep. 2015;64(4):93-94.

3. Kim DK, Bridges CB, Harriman KH. Advisory Committee on Immunization Practices (ACIP), ACIP Adult Immunization Work Group. Advisory Committee on Immunization Practices Recommended Immunization Schedule for Adults Aged 19 or Older-United States, 2015. Morbid Mortal Wkly Rep. 2015;64(4);91-92.

4. London NJ, Garg SJ, Moorthy RS, Cunningham ET. Drug-induced uveitis. J Ophthalmic Inflamm Infect. 2013;3(1):43.

5. Fraunfelder FW, Suhler EB, Fraunfelder FT. Hepatitis B vaccine and uveitis: an emerging hypothesis suggested by review of 32 case reports. Cut Ocul Toxicol. 2010;29(1)26-29.

6. Escott S, Tarabishy AB, Davidorf FH. Multifocal choroiditis following simultaneous hepatitis A, typhoid, and yellow fever vaccination. Clin Ophthalmol. (Auckland, NZ). 2013;7:363-365.

7. Stangos A, Zaninetti M, Petropoulous L, Baglivo E, Pournaras C. Multiple evanescent white dot syndrome following simultaneous hepatitis A and yellow fever vaccination. Ocul Immunol Inflamm. 2006;14(5):301-304.

8. Fine L, Fina A, Cunningham ET Jr. Multiple evanescent white dot syndrome following hepatitis A vaccination. Arch Ophthalmol. 2001;119(12):1856-1858.

9. CDC Advisory Committee on Immunization Practices. A comprehensive immunization strategy to eliminate transmission of hepatitis B virus infection in the United States. Morbid Mortal Wkly Rep. 2005;54(RR-16):1-32.

10. Wells MB, Garg S. Bilateral panuveitis after influenza vaccination. Retinal Cases Brief Rep. 2009;3(4):386-387.

11. Blanche P, Decrette C, Sicard D. Development of uveitis following vaccination for influenza. Clin Infect Dis. 1994;19(5):979.

12. Knopf HL. Recurrent uveitis after influenza vaccination. Ann Ophthalmol. 1991;23(6):213-214.

13. Islam SM, Tabbara KF. Causes of uveitis at The Eye Center in Saudi Arabia: a retrospective review. Ophthalmol Epidemiol. 2002;9(4):239-249.

14. Spratt A, Key T, Vivian AJ. Chronic anterior uveitis following bacilli Calmette-Guerin vaccination: molecular mimicry in action? J Pediatr Ophthalmol Strabismus 2008;45(4):252-253.

15. Garip A, Diedrichs-Mohring M, Thurau SR, Deeg CA, Wildner G. Uveitis in a patient treated with bacilli-Calmette Guerin: possible antigenic mimicry of mycobacterial and retinal antigens. Ophthalmology 2009;116(12):2467-2462. e2451-2452.

16. Islam SM, El-Sheikh HF, Tabbara KF. Anterior uveitis following combined vaccination for measles, mumps and rubella (MMR): a report of two cases. Acta Ophthalmol Scand. 2000;78(5):590-592.

17. Sedaghat M, Zarei-Ghanavati S, Shokoohi S, Ghasemi A. Panuveitis and dermal vasculitis following MMR vaccination. East Mediterr Health J. 2007;13(2):470-474

\section{Disclosure}

None reported. MM 